\title{
Obtención de efectos físico-ópticos para la decoración de baldosas cerámicas
}

\author{
M. J. CABRERA, V. MONTINS, D. SOLSONA, J. M. SALA. \\ Vidres S.A. Ctra. de Onda, 12. 12540 Vila-real. Castellón
}

Este trabajo ha obtenido el premio Alfa de oro en la Feria Internacional de Cerámica CEVISAMA 2012

\begin{abstract}
En el presente trabajo se ha desarrollado una gama de productos para la decoración de baldosas cerámicas caracterizados porque tras su aplicación sobre un sustrato cerámico y posterior cocción del mismo, se genera una estructura de microgrietas en dichos recubrimientos que provoca la aparición de un efecto iridiscente, multicolor, que confiere a la pieza cerámica características estéticas diferenciales, con un aspecto similar al que se puede observar en el mineral "cuarzo iris o cuarzo rainbow".

Se realiza un análisis del estado de la técnica y se profundiza en el estudio y caracterización de los fenómenos físico-ópticos responsables de dichos efectos en estos minerales, con el objetivo de determinar y modelizar como se produce el "efecto multicolor" o efecto iridiscente, fijar la causa de dicho efecto , determinar el fenómeno físico-óptico que lo provoca, analizar y entender los mecanismos fenomenológicos que lo causan para intentar extrapolar dichos efectos a nuestros materiales cerámicos.
\end{abstract}

\section{Palabras clave: Baldosas cerámicas, estructuras de microgrietas, cuarzo iris, cuarzo rainbow}

Physical-optical effects obtention for the ceramic tiles decoration.

This paper presents a range of products for the ceramic tiles decoration, characterized for a microcracks structure after its application on a ceramic substrate and subsequent firing. This structure origins a multicoloured iridescent effect that confers to the ceramic tile differential aesthetics characteristics, with a final aspect similar to the "rainbow quartz" or "iris quartz".

An analysis of the state of the art is made as well as a deepening in the study and characterization of the physicaloptical phenomena responsible of that kind of effects in these minerals, with the aim of determining and modelling the "multicoloured effect" or iridescent effect, the cause of this effect, the physical-optical phenomenon that produces it, and the analysis and knowledge of the phenomenological mechanisms that origins it, so that we can try the extrapolation of that effects wit our ceramic materials.

Keywords: ceramic tiles, microcracks structure, rainbow quartz, iris quartz

\section{INTRODUCCIÓN, ANTECEDENTES Y OBJETIVO DEL PROYECTO}

En la industria cerámica, el recubrimiento superficial de los soportes cerámicos confiere a las piezas las decoraciones o características técnicas que permiten una diferenciación y diversificación de los productos y poder dotarlos de gran valor añadido.

El resultado de la decoración de las baldosas cerámicas es lo que vemos, lo que nos atrae y lo que nos permite diferenciar estéticamente unas piezas de otras. En la mayoría de ocasiones, lo que vemos, el aspecto final de la baldosa es el resultado de procesos de cristalización de fases que nos permiten apreciar diferentes acabados: aspecto mate, brillo, opacidad, lustre, etc. Pero hay otros fenómenos no debidos a la formación de cristales, ni a la introducción de tintas o pigmentos en las decoraciones, sino debidos a la interacción de la luz con los materiales. Estos fenómenos ópticos, su estudio y comprensión, sus mecanismos de obtención y potenciación, muy utilizados en otros sectores tales como el sector de las pinturas y plásticos, pueden permitirnos abrir nuevas líneas de investigación para continuar dotando a nuestro sector de nuevas propuestas decorativas que permitan seguir diferenciando y diversificando nuestros azulejos.

El objetivo de este trabajo ha sido determinar y modelizar como se produce el "efecto multicolor" o efecto iridiscente en determinados minerales, fijar la causa de dicho efecto, determinar el fenómeno físico-óptico que lo provoca, analizar y entender los mecanismos fenomenológicos que lo causan para intentar extrapolar dichos efectos a nuestros materiales cerámicos y poder desarrollar nuevos recubrimientos que presenten propiedades ópticas determinadas.

Conocer con exactitud el fenómeno físico-óptico que genera el efecto final, como se genera, cuales son los parámetros dentro del proceso productivo que hay que determinar para reproducir exactamente el mismo efecto iridiscente en cada muestra, controlar para saber como modificarlo y alterar para 
conseguir el efecto según requisitos estéticos. Entender el fenómeno que causa el efecto multicolor es fundamental para poder comprender las modificaciones que se pueden hacer en un material para conseguir distintos efectos a partir del mismo fenómeno, así como para conocer cuáles son los límites; es decir, bajo que condiciones es posible generar el efecto y bajo cuales no.

Así pues se ha partido de la realización de una búsqueda bibliográfica de minerales existentes en la naturaleza que puedan tener diferentes cromatismos dependiendo de cómo incida de la luz estudiando el porqué de este efecto para intentar extrapolar su obtención en las superficies de las baldosas cerámicas.

La iridiscencia es uno de los fenómenos ópticos más bellos de algunos minerales, caracterizado como la propiedad de ciertas superficies en las cuales el tono de la luz varía de acuerdo al ángulo desde el que se observa dicha superficie. Este fenómeno óptico no se debe a la composición química ni a la estructura cristalina sino a la interacción de la luz con ciertas inclusiones o defectos estructurales en los mismos.

Uno de los fenómenos potencialmente causante de la aparición de este tipo de efectos puede ser la dispersión de la luz, fenómeno que provoca, por ejemplo la descomposición de la luz blanca en sus distintos colores cuando esta atraviesa un prisma, o la aparición del arcoíris los días lluviosos. Este fenómeno está ligado a la refracción de la luz y las diferencias de índice de refracción entre los distintos medios materiales que atraviesa la luz.

Otro fenómeno que puede estar involucrado en la aparición del efecto iridiscente es la interferencia de la luz. Este fenómeno es el causante, por ejemplo, del efecto iridiscente que se aprecia en las pompas de jabón o en las conchas de algunos moluscos. Este fenómeno se produce por las interferencias constructivas y destructivas que se producen en las estructuras bicapa o multicapas semitransparentes al interaccionar la luz reflejada por las sucesivas capas de la estructura.

El último fenómeno que puede estar causando este efecto es la difracción de la luz. Este fenómeno físico-óptico causado fundamentalmente por las irregularidades que la luz encuentra en su trayectoria, por ejemplo diminutas protuberancias $\mathrm{u}$ oquedades, que han de ser del tamaño similar a la longitud de onda de la luz que se difracta. Este fenómeno es el causante de la descomposición de la luz blanca en los distintos colores del espectro visible que se puede apreciar en la cara espejada de los discos compactos (CD).

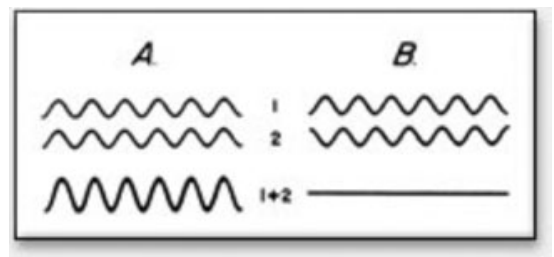

Constructive vs. destructive interference: light waves 1 and 2 produce constructive reinforcement if they are in phase (A). Crests align with crests and troughs align with troughs, reinforcing one another. Destructive cancellation is observed when they are out of phase (B). The crests of wave 1 align with the troughs of wave 2, canceling one another.
La luz blanca pasa a través de pequeños espacios como poros o hendiduras, o atraviesa finas capas de material con diferente índice de refracción que actúan como primas separando la luz blanca en todos los colores del espectro.

Se produce cuando la luz entra en un medio en el cual se producen múltiples reflexiones debido a la presencia de muchas superficies semitransparentes, donde los cambios de fase e interferencias de las reflexiones modulan la luz por la amplificación o atenuación de las diferentes frecuencias.

Las longitudes de onda interfieren unas con otras causando la iridiscencia. Cuando dos ondas ópticas se encuentran el espacio, si estas tienen la misma longitud de onda pueden interactuar reforzándose si están en fase o eliminándose. Si se encuentran de modo que la cresta de una coincida con la cresta de la otra onda se dice que las ondas están en fase y se obtiene una máxima amplitud como suma de amplitudes de las ondas individuales produciéndose una interferencia constructiva. El efecto opuesto, la interferencia destructiva se presenta cuando la cresta de una onda coincide con el valle de la otra.

Este fenómeno de iridiscencia está presente en muchos minerales tipo Labradurita, ágatas, ópalos, etc.

En algunas patentes se citan diferentes metodologías para obtener el efecto decorativo tipo "iris" o "rainbow" en varios tipos de materiales. Entre ellas podemos citar la patente JP200034145 para producir este tipo de efectos en sustratos en cuya superficie se sitúan esferas de vidrio las cuales se recubren de un film de dióxido de titanio.

En la patente JP7149585 se obtienen baldosas con este efecto decorativo mediante pulido de ciertas zonas de las baldosas y recubriendo dichas zonas pulidas con una resina metálica.

En la patente JP5269953 se describe un proceso para obtener materiales con este efecto a partir de un film transparente al que se adicionan partículas de oro, plata, aluminio, mica y cerámica seguidas de una capa de adhesivo.

En la patente CN101973711 se describe la preparación de un vidriado a partir de un medio orgánico junto con un compuesto metálico soluble y un adhesivo orgánico aplicable a cerámica, porcelana y otros sustratos para obtener una fina capa metálica con efectos estéticos tipo iridiscencias o "rainbow".

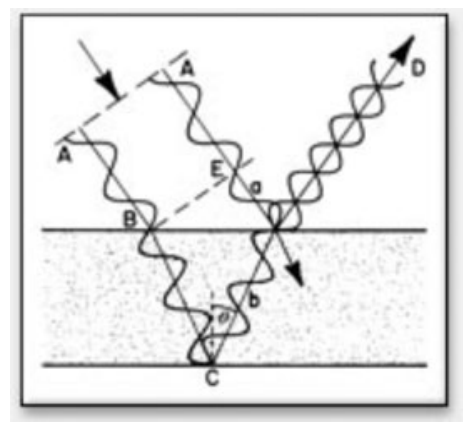

Interference of light beams reflected from the front and back surfaces of a thin parallel film. This effect can be seen in soap bubbles. As either the angle, the thickness of the film, or the wavelength changes, the wavelength and the color of light produced by constructive interference changes. 
En la patente KR20040080628 este tipo de efectos se consiguen sobre baldosas cerámicas con la ayuda de un plasma equipado con electrodos de titanio.

Hay otros casos en los que la iridiscencia realmente revela un defecto, la presencia de microscópicas grietas o hendiduras responsables por ejemplo de la belleza del cuarzo conocido como "cuarzo iris" o "cuarzo rainbow".

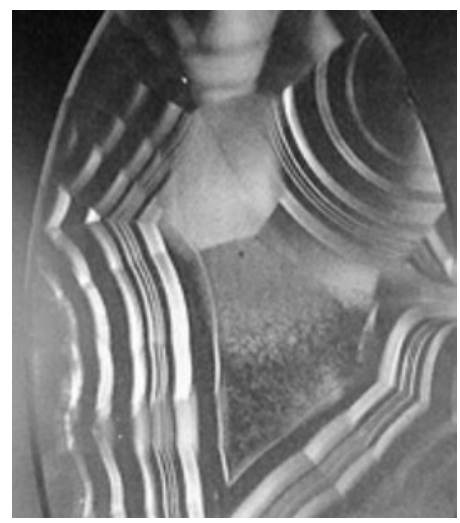

El cuarzo es el mineral más difundido en la naturaleza y está formado por óxido de silicio. Cuando su variedad incolora (cristal de roca) presenta una difusa y fina fracturación puede aparecer una típica iridiscencia debido a fenómenos de interferencia entre la luz incidente y las burbujitas de gas o de líquidos presentes en las propias fracturas. Este mineral, si la fracturación es en particular muy marcada, puede comportarse como un prisma óptico que descompone la luz en sus colores básicos.

Los cristales de cuarzo iris o cuarzo "rainbow" pueden presentar fisuras o microgrietas generalmente producidas por estrés mecánico. Debido a que se trata de fracturas concoidales, dichas fisuras son curvas y con anchura variable. La fractura concoidea o concoidal es un tipo de rotura propia de materiales frágiles, de composición homogénea, pero amorfa, que al trocearse no siguen planos naturales de separación.

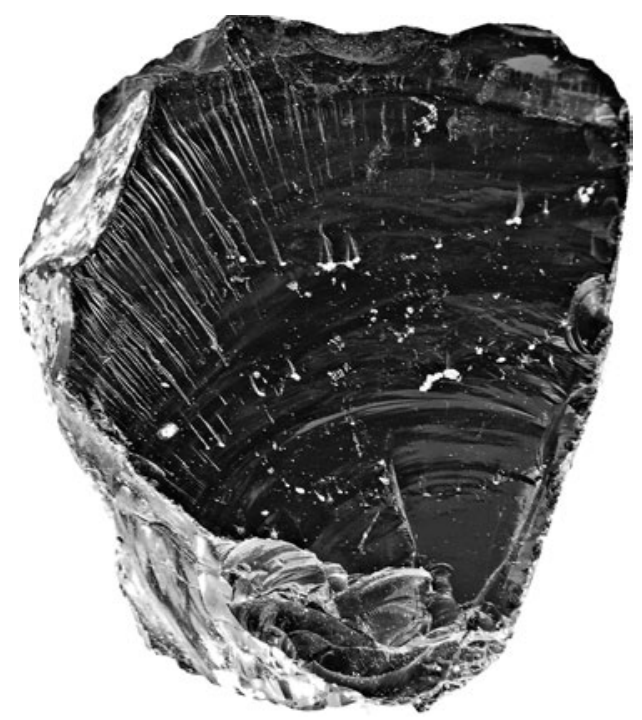

La fractura concoidea, se produce a través de una superficie equipotencial que no es plana, una onda curva, y que, además, cambia paulatinamente a medida que se propaga.

El nacimiento es la parte donde el material recibe la tensión que va a provocar la fractura. Esta zona recibe el nombre de superficie elíptica de Hertz, y a partir de ella, como si de un epicentro se tratase, se desarrollan ondas de vibración que rompen el material y que son reflejadas una y otra vez por todas las caras del sólido hasta que, por fin, confluyen en una única superficie de fractura. Estas ondas tienen forma de parábolas anchas y cortas cuyo origen es tangente (parábolas homofocales). A medida que la superficie de fractura se propaga, las parábolas cambian de dirección.

Las fisuras son generalmente muy finas y a menudo rellenas de aire. La luz que atraviesa la fisura puede ser parcialmente reflectada en ambos lados o caras de la fisura. Si la anchura de dicha fisura es del mismo orden de magnitud que la de la longitud de onda de la luz incidente, los rayos reflectados producen interferencia (partes del espectro se eliminan y la luz reflectada es multicolor). El color depende de la anchura de la grieta y del ángulo con el que la luz atraviesa dicha grieta. Dado que la anchura de estas fisuras varía gradualmente se producen reflexiones de diferentes colores tipo arco iris produciendo el fenómeno conocido como iridiscencia.

\subsection{Formación de microgrietas en los minerales}

En general, en los minerales, la mayor parte de las microgrietas se producen en zonas donde existen defectos previos, como límites de grano, inclusiones, poros, maclas, dislocaciones u otras microgrietas previas. En el nivel atómico, la formación y crecimiento de las microgrietas supone la rotura de enlaces atómicos.

El esfuerzo que provoca la formación de microgrietas puede ser tectónico, gravitatorio o térmico. El calentamiento y enfriamiento provocan que minerales vecinos se expandan o contraigan de manera diferente (en función del coeficiente de expansión térmica) provocando la aparición de puntos de concentración de esfuerzos. Si esta concentración es suficientemente grande, se formará una microgrieta en el punto de concentración. En granitos, por ejemplo, la diferencia en los coeficientes de expansión térmica del cuarzo y el feldespato es la causa principal de la aparición de microgrietas.

El enterramiento y la erosión también generan microgrietas, no sólo debido a los cambios de temperatura asociados con los cambios de profundidad, sino también por el efecto de carga y descarga gravitatoria. Las microgrietas también se forman como resultado de los esfuerzos tectónicos, sobre todo cerca de microgrietas previas y contactos entre granos. Cuando la deformación es semi-frágil o dúctil, la concentración de esfuerzos se puede producir cuando la propagación de dislocaciones encuentra un obstáculo como un borde de grano, una cavidad u otra dislocación. La microgrieta se forma cuando el movimiento de la dislocación no puede acomodarse por deformación elástica de la red cristalina.

\subsection{Formación de microgrietas en materiales cerámicos}

Adentrándonos en el estudio de cómo se pueden producir estructuras con microgrietas en los materiales cerámicos, M. W. Barsoum en "Spontaneous Microcracking of Ceramics" 
Chapter 13. Fundamentals of Ceramics, Institute of Physics Publishing, 2003 pone de manifiesto que en materiales cerámicos, las microgrietas se inician debido a cambios de temperatura durante el enfriamiento como consecuencia de un aumento del estrés residual. Dicho estrés es causado fundamentalmente por la expansión térmica anisotrópica de los materiales y por las transformaciones de fase que van acompañadas a cambios de volumen.

\subsubsection{MICROGRIETAS DEBIDAS AL ESTRÉS RESIDUAL PROVOCADO POR TRANSFORMACIONES O CAMBIOS DE FASE.}

Debido a los cambios de temperatura durante la cocción, se producen modificaciones en la estructura cristalina de los materiales, variando las distancias interatómicas y las amplitudes de las vibraciones atómicas, la estructura cristalina que presentaba inicialmente el material ya no es estable y pasa a otra estructura de mayor estabilidad.

Dichas transformaciones conllevan a reordenamiento de átomos asociados a cambios de volumen.

Los materiales que tienen la misma composición química pero diferente estructura cristalina se denominan polimorfos y el cambio de una estructura a otra transformación polimórfica. Dependiendo del tipo de cambios que ocurren en el cristal se pueden distinguir dos tipos de transformaciones, por desplazamiento y por reordenación o difusionales (reconstructivas).

En el caso de las transformaciones por desplazamiento todos los átomos del cristal se desplazan al mismo tiempo, una distancia cuya magnitud no es superior a la distancia interatómica, lo que se traduce en una alteración de toda la estructura cristalina.

Como consecuencia existe una variación en los ángulos de enlace pero nunca tiene lugar la rotura de los mismos. Estas transformaciones tienen lugar a una temperatura definida, ocurren rápidamente y están acompañadas de una variación de volumen ("Transformaciones de fase en materiales cerámicos". Paloma Fernández Sánchez. Departamento de Física de Materiales, Físicas, UCM)

Como ejemplo de este tipo de transformaciones tenemos la circona tetragonal que sufre una transformación de fase pasando de simetría tetragonal a monoclínica.

El óxido de circonio tiene una transformación de fase martensítica cuando la forma polimórfica tetragonal metaestable pasa a la monoclínica estable, lo que ocurre a una temperatura de aproximadamente $1170-1180{ }^{\circ} \mathrm{C}$ implicando un cambio de forma y de volumen.

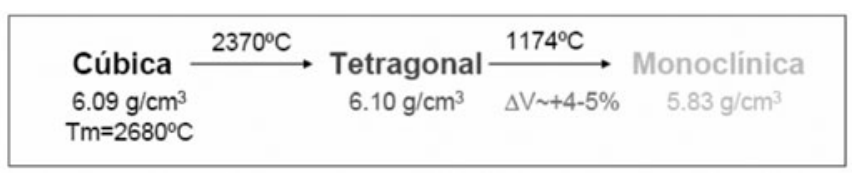

Al enfriarse los granos de óxido de circonio fundido, estos pasan por las siguientes fases cristalinas: cúbica $(\mathrm{k})$, tetragonal (t) y monoclínica (m). La transformación de fase $\mathrm{ta}$ m es una transformación sin difusión (transformación martensítica). Esta transformación se asocia a un incremento de volumen del 3 al $5 \%$.
Esto significa que el $\mathrm{ZrO}_{2}$ sufre un incremento de volumen dando lugar a microfisuras asociadas a este incremento. Añadiendo otros óxidos, esta transformación de fases puede desplazarse a temperaturas inferiores.

Si el tamaño de grano es bastante pequeño la resistencia de los granos adyacentes impiden que tenga lugar la transformación, previniendo la necesaria expansión de volumen.

Otro ejemplo de transformación polimórfica lo encontramos en la sílice $\mathrm{SiO} 2$,

produciéndose diferentes transformaciones tanto de desplazamiento como de reconstrucción dependiendo de la temperatura.
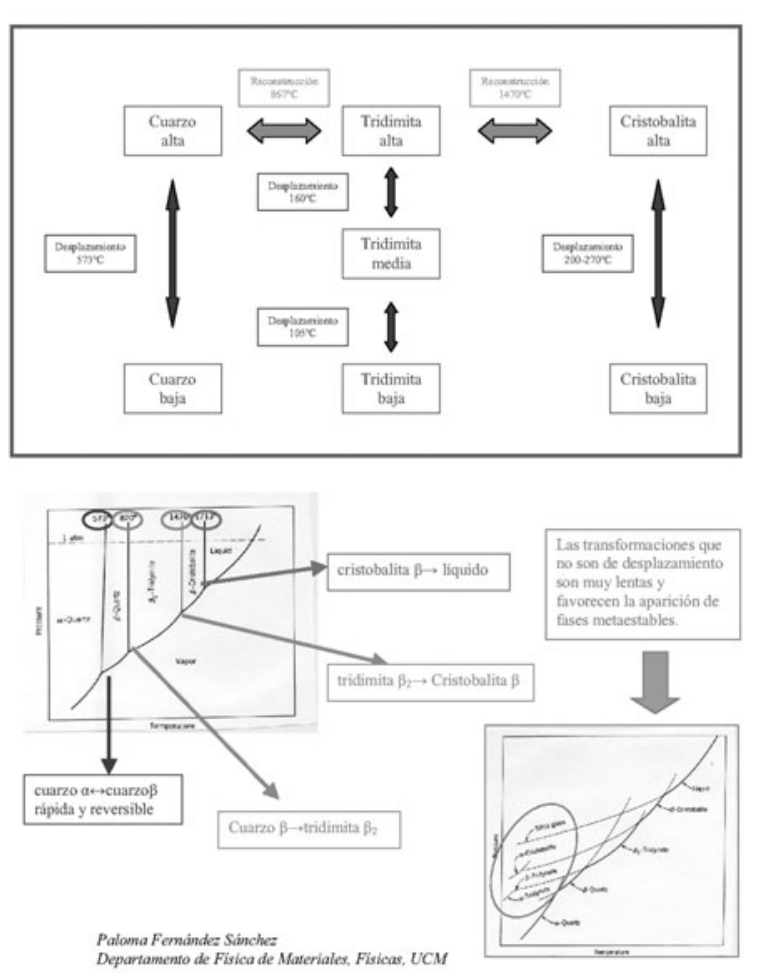

\subsubsection{MICROGRIETAS DEBIDAS A LA EXPANSIÓN TÉRMICA ANISOTRÓPICA.}

Las microgrietas se producen alrededor de los límites o fronteras entre granos, a partir de un tamaño de grano crítico y son debidas a las diferencias de coeficiente de expansión térmica entre materiales.

Dichas microgrietas son los resultados de esfuerzos internos entre diferentes granos, inducidos por deformaciones incompatibles de las transformaciones de fase y de la anisotropía en la expansión térmica.

Se han realizado estudios sobre el origen de microgrietas espontáneas debidas a la expansión o dilatación térmica anisotrópica de los materiales y su efecto en las propiedades físicas de dichos materiales. En materiales cerámicos policristalinos la dilatación o expansión térmica anisotrópica de los gránulos incrementa el estrés residual durante la etapa de enfriamiento y dicho estrés residual da lugar a la formación espontánea de microgrietas, inicialmente alrededor 
de la frontera o los límites entre gránulos. ("Microcraking in Ceramics Induced by Thermal Expansion or Elastic Anisotropy “, V. Tvergaard and J.W. Hutchinson. Journal of the American Ceramic Society, Vol. 71, No. 3 March 1988).

Asumiendo que los diferentes granos son elásticamente isotrópicos en un agregado policristalino, se deduce que la microgrieta es debida principalmente al estrés residual que se produce durante la etapa de enfriamiento de los materiales. Para la mayoría de materiales cerámicos hay un límite de temperatura por debajo de la cual y debido a procesos de relajación de estrés se pueden producir microgrietas.

Las tensiones térmicas desarrolladas en una pieza de un material sometido a un cambio brusco de temperatura son proporcionales al producto del coeficiente de expansión térmica, y del módulo de elasticidad.

La teoría que describe el sistema de tensiones alrededor de una partícula aislada en un medio isótropo infinito, debido a las diferencias entre los coeficientes de expansión térmica de la partícula y la matriz, ha sido bien establecida. Una partícula esférica estará sometida a una presión $\mathrm{P}$ que, para bajas concentraciones de partículas, esta dada por la ecuación:

$$
P=\frac{\Delta \alpha \cdot \Delta T}{\frac{\left(1+v_{m}\right)}{2 \cdot E_{m}}+\frac{\left(1-2 v_{p}\right)}{E_{p}}}
$$

donde $\Delta \alpha$ es la diferencia entre los coeficientes de expansión de la partícula, $\mathrm{p}$, y matriz, $\mathrm{m}, \Delta \mathrm{T}$ es la diferencia entre la temperatura en la cual ya no existe relajación de tensiones y la temperatura considerada, $v_{\mathrm{m}^{\prime}} v_{\mathrm{p}}$ y $\mathrm{E}_{\mathrm{m}^{\prime}} \mathrm{E}_{\mathrm{p}}$ son el coeficiente de Poisson y el módulo de elasticidad de la matriz y las partículas, respectivamente.

El signo de las tensiones creadas depende de si la contracción de las partículas durante el enfriamiento es mayor o menor que la de la matriz. En materiales con $\alpha_{p}>\alpha_{m}$ la partícula esta sometida a tracción y las microgrietas se forman alrededor de la partícula. En materiales con $\alpha_{\mathrm{p}}<\alpha_{\mathrm{m}}$ la partícula esta sometida a compresión y se desarrollan microgrietas radiales. Este tipo de microgrietas es más perjudicial para los materiales en términos de tensión de fractura ya que pueden llegar a coalescer y dar lugar a defectos en la matriz.

Aunque la ecuación muestra que la magnitud de las tensiones térmicas es independiente del tamaño de partícula, experimentalmente se ha observado que el microagrietamiento solamente ocurre a partir de un cierto tamaño de las partículas.

El módulo de elasticidad, coeficiente de expansión térmica y conductividad térmica son propiedades variables que dependen fundamentalmente del tamaño de grano de las partículas que conforman el material. En particular, para cada material existe un tamaño de grano crítico, $\mathrm{d}$ cr, por debajo del cual no tiene lugar la formación de microgrietas.

La dependencia de la formación de microgrietas con el tamaño de grano puede explicarse, y ha sido estudiada por distintos autores, en base a criterios energéticos, ej. N. Claussen, B. Mussler and M.V. Swain "Grain-size dependence of fracture energy in ceramics" J. Am. Ceram. Soc., 65 C14-16 (1982).

El tamaño crítico de las partículas puede ser estimado por un criterio de balance energético del mismo tipo que el utilizado por Cleveland y Bradt (J. Amer. Cer. 61:478
(1978)) para el cálculo del $\mathrm{d}_{\mathrm{cr}}$ en los materiales no cúbicos: para que se cree una microgrieta, la energía elástica de deformación acumulada en la partícula, $E$, tiene que igualar a la energía necesaria para crear las dos nuevas superficies de la microgrieta, $2 \cdot \gamma_{\mathrm{f}}$,

Si se asume que se forma una microgrieta alrededor de la partícula. Para partículas esféricas la ecuación vendrá dada por:

$$
E_{t o t a l}=-E \cdot \frac{4}{3} \pi \cdot r^{3}+2 \cdot y_{f} \cdot 4 \cdot \pi \cdot r^{2}
$$

donde $\mathrm{r}$ es el radio de la partícula, $2 \cdot \mathrm{r}=\mathrm{d}_{\mathrm{cr}}$ a partir del cual se formarán microgrietas.

Específicamente, hay un tamaño de partícula "crítico" por debajo del cual la microgrieta no se produce y por encima del cual ocurre de forma espontánea. Materiales con tamaño de partícula fino no presentan microgrietas dado que el estrés residual debido a la expansión térmica anisotrópica no es suficiente para nuclear las microgrietas.

En segundo lugar, las microgrietas que se generan espontáneamente se producen principalmente alrededor de los límites o frontera entre gránulos.

De acuerdo a estos estudios, los parámetros más significantes que afectan al tamaño crítico de grano para que se produzcan las microgrietas son por una parte la diferencia entre coeficientes de dilatación térmica y el descenso de temperatura.

Otros autores no hacen el supuesto de asumir que los gránulos presentan una elasticidad isotrópica (constante en cualquier dirección) sino anisotrópica (variación de características o propiedades según la dirección) eincluyen este efecto anisotrópico en los modelos de estudio. Dependiendo de la combinación entre elasticidad y anisotropía térmica y de la orientación de los gránulos en el agregado policristalino el estrés puede mejorar o reducirse en el límite de grano. Si la anisotropía elástica amplifica el estrés residual resultante por las contracciones térmicas, el material puede fracturarse a un tamaño de partícula menor que el que se produce teniendo en cuenta exclusivamente la anisotropía por dilatación térmica.

Por otra parte existen numerosos estudios en los que mediante modelos matemáticos se concluye que el inicio de las microgrietas depende de la geometría de los gránulos y de las orientaciones relativas de los ángulos de los mismos.

Por ejemplo, en materiales cerámicos tipo porcelana, durante el enfriamiento, el bajo coeficiente de expansión térmica de la fase vítrea comparado con el de las partículas de cuarzo causa tensiones radiales que pueden dar lugar a la formación de microgrietas entre estos dos componentes. En estos casos, cuando disminuye el tamaño de las partículas de cuarzo, la facilidad de producirse microgrietas aumenta, se desplaza hacia valores de temperatura menor, pero si el tamaño de las partículas es demasiado fino no se observan dichas microgrietas. ("Structure dependence of thermally induced microcracking in porcelain studied by acoustic emission". G. Kirchhoff, W.Pompe, H.A. Bahr. Journal of Materiales Science 17 (1982) 2809-2816. )

Todas estas teorías y modelos matemáticos han sido puestos en práctica en algunos trabajos, con diferentes objetivos.

Por ejemplo en algunos trabajos se pretende la generación de microfisuras con el objetivo de poder cortar vidrios 
(WO2006045130) o en otros se describe un procedimiento para la realización de una zona de rotura prevista en un cuerpo de vidrio creando un anillo de rotura mediante la generación en dicha zona de microfisuras por exposición a radiación láser (ES2168148).

En otros trabajos se asume que las microestructuras que tienen microgrietas residuales son útiles en las situaciones que se requiere resistencia al choque térmico y aumentan la resistencia a la fractura de dichos materiales.

Así por ejemplo, en la patente WO2011150145 se describe un cuerpo cerámico poroso con estructura de nido de abeja diseñado para ser utilizado como filtro de gases de combustión en automóviles. Dicho cuerpo cerámico es una matríz de cordierita que se somete a tratamientos térmicos para provocar microgrietas controladas y cambios en el coeficiente de expansión del material con el objetivo de aumentar la resistencia a la rotura de estos materiales.

En la patente US4775648 se describen cuerpos cerámicos sinterizados y calcinados para ser utilizados como materiales refractarios en los que se busca un sistema de microgrietas uniformemente distribuido en dichos cuerpos cerámicos.

En la patente US4506024 se describe el proceso de producción de cuerpos cerámicos utilizados en la construcción de piezas de ingeniería que van a ser sometidas a altas temperaturas para las cuales se necesita un alta densidad y elevada fuerza de rotura.

El objetivo principal del presente trabajo consiste en el desarrollo de materiales para la decoración de baldosas cerámicas a partir de efectos físico-ópticos y en concreto que presenten una interacción con la luz similar a la que presenta el mineral cuarzo iris o cuarzo rainbow para poder simular el efecto iridiscente, multicolor de estos minerales, para lo cual, mediante composiciones y procesos se buscará la obtención de una estructura de microfisuras para intentar simular dichos efectos.

\section{DESARROLLO DEL PROYECTO}

Para alcanzar el objetivo propuesto y tras una revisión bibliográfica de los fenómenos que dan lugar a este tipo de efectos de iridiscencia presentes en el cuarzo iris o cuarzo rainbow será necesario buscar las composiciones adecuadas para producir el tipo de microfisuras deseadas.

El estudio englobará el conocimiento de su mecanismo de formación y variables que afectan a la formación y crecimiento de dichas microgrietas y el intento de conseguir reproducir estas formaciones de microgrietas en los materiales cerámicos a formular.

Es importante resaltar que la formación, localización y control del tamaño de las microgrietas formadas entre las partículas y la matriz o esmalte cerámico será importante, no solo para conseguir los efectos ópticos deseados, sino también para evitar que la presencia de microgrietas inducidas por tensión o residuales puede dar lugar a una reducción significativa en la resistencia a la fractura. Debido a la creciente resistencia a la fractura, las microestructuras que tienen microgrietas residuales son útiles en las situaciones que se requiere resistencia al choque térmico. Estos materiales se deben procesar cuidadosamente para causar solamente el desarrollo limitado de la microgrieta.
El desarrollo del proyecto se ha centrado en diferentes etapas:

Desarrollo de partículas de diferentes coeficientes de dilatación, para poder ser introducidas en esmaltes cerámicos. Control del tamaño de partícula de los gránulos desarrollados.

Estudio y control de la morfología de las partículas desarrolladas.

Desarrollo de los esmaltes cerámicos más adecuados para la obtención de los efectos deseados.

Estudio de diferentes ciclos de cocción y transformaciones de fase que se producen durante la cocción de los materiales a desarrollar.

Estudios de diferentes técnicas de decoración, formatos, diseños gráficos y propuestas decorativas.

Caracterización física de las piezas obtenidas.

Estabilidad, reproducibilidad y posible escalado industrial.

\section{RESULTADOS Y DISCUSIÓN}

Dado que los coeficientes de dilatación de estas partículas dependen de la composición de dichas partícula, se buscaron las composiciones adecuadas, para poder ser introducidas en esmaltes cerámicos de forma que aunque dichas partículas presenten diferentes coeficientes de dilatación, la influencia sobre el conjunto del esmalte cerámico sea mínima para permitir un buen acoplamiento entre los coeficientes de dilatación de los esmaltes, engobes y soporte cerámico sobre el que se aplicarán. Los soportes cerámicos presentan un coeficiente de dilatación bastante estable definido por las materias primas que los componen. Los esmaltes y engobes cerámicos generalmente presentan coeficientes de dilatación adecuados a dichos soportes y una brusca modificación de los mismos daría lugar a una serie de defectos indeseados en las piezas cerámicas tales como curvaturas de las piezas, cuarteo, desconchados, etc. Esto hace fundamental que las partículas o gránulos a introducir en las nuevas formulaciones no modifiquen demasiado los coeficientes de dilatación de los esmaltes cerámicos.

Para poder establecer el tamaño de partícula óptimo como parámetro crítico a la hora de conseguir el objetivo del proyecto, se prepararon cinco muestras de partículas de idéntica composición química y morfología cuyos valores granulométricos obtenidos mediante difracción láser (Coulter) se muestran en la siguiente tabla:

\begin{tabular}{|c|c|c|c|c|}
\hline Referencia & Mean & d10 & d50 & d90 \\
\hline IN-31/110-2 & $11,21 \mu \mathrm{m}$ & $0,792 \mu \mathrm{m}$ & $7,974 \mu \mathrm{m}$ & $\begin{array}{c}27,82 \\
\mu \mathrm{m}\end{array}$ \\
\hline $\mathrm{IN}-31 / 110-3$ & $41,97 \mu \mathrm{m}$ & $26,59 \mu \mathrm{m}$ & $40,51 \mu \mathrm{m}$ & $\begin{array}{c}60,18 \\
\mu \mathrm{m}\end{array}$ \\
\hline $\mathrm{IN}-31 / 110-4$ & $103,6 \mu \mathrm{m}$ & $82,46 \mu \mathrm{m}$ & $102,6 \mu \mathrm{m}$ & $\begin{array}{c}126,9 \\
\mu \mathrm{m}\end{array}$ \\
\hline $\mathrm{IN}-31 / 110-5$ & $195,3 \mu \mathrm{m}$ & $137,0 \mu \mathrm{m}$ & $190,1 \mu \mathrm{m}$ & $\begin{array}{c}262,0 \\
\mu \mathrm{m}\end{array}$ \\
\hline $\mathrm{IN}-31 / 110-6$ & $320,7 \mu \mathrm{m}$ & $299,8 \mu \mathrm{m}$ & $315,5 \mu \mathrm{m}$ & $\begin{array}{c}422,2 \\
\mu \mathrm{m}\end{array}$ \\
\hline
\end{tabular}


Estas partículas se mezclaron con un micronizado cerámico y se aplicaron en forma de serigrafía sobre piezas de gres porcelanico esmaltado. Tras la cocción de las piezas no se observó ningún efecto óptico en las muestras referenciadas como IN-31/110-2 y IN-31/110-3 y su observación al microscopio indicó que no existía presencia de microgrietas en dichas piezas.

En cambio las muestras referenciadas como IN-31/1104 y IN-31/110-5, daban lugar a efectos ópticos en la pieza final tipo iridiscencias o efecto multicolor, este efecto óptico era más acentuado (mayor efecto iridiscente y multicolor) en la muestra referenciada como IN-31/110-4. Mediante el microscopio estereoscópico se pudo observar la presencia de microgrietas en estas piezas, de mayor tamaño en la muestra referenciada como IN-31/110-5.

En cuanto a la muestra IN-31/110-6 aunque también se observaba la presencia de microgrietas, debido al mayor tamaño de las mismas, el efecto óptico obtenido no era el deseado, minimizándose considerablemente el efecto iridiscente.

Así pues, tras el estudio de partículas con diferentes granulometrías se pudo concluir que para una misma composición química de partículas existía un límite tanto inferior como superior de tamaño de partícula óptimo para la obtención del efecto, siendo dicho intervalo el comprendido entre 60 y 350 micras.

Por otra parte y dado que en la bibliografía se especifica que las microgrietas que se generan espontáneamente se producen principalmente alrededor de los límites o frontera entre gránulos se realizaron ensayos con diferentes porcentajes por unidad de área de las partículas desarrolladas. Tras cocción y observación al microscopio se comprobó que existía una cantidad mínima de porcentaje por unidad de área de partículas nucleadoras o formadoras de las microgrietas por debajo de la cual no se generan y por tanto no se obtiene el efecto óptico de iridiscencia o efecto multicolor.

Con el fin de evaluar la posible transformación de fase de las partículas nucleadoras o formadoras de microgrietas durante la etapa de cocción, se realizó identificación de las fases cristalinas presentes mediante difracción de rayos- $X$ y posterior análisis de las fases obtenidas en base al banco de datos de difracción de polvo (Powder Difraction File) del ICDD-JCPDS (International Centre for Difraction DataJoint Cometee of Powder Diffraction Standards, 2010) de las muestras en polvo de las partículas antes de cocción y de la zona de la pieza con presencia de microgrietas y por tanto de efecto iridiscente, multicolor obtenido tras la cocción de la pieza. Los resultados obtenidos confirmaron la transformación de fase de dichas partículas durante el proceso de cocción de la pieza.

Los productos desarrollados, se han adaptado a varias de las técnicas de aplicación utilizadas habitualmente en el sector de fabricación de baldosas cerámicas decoradas, obteniéndose piezas aplicadas vía húmeda en forma de esmaltes a campana y discos, piezas con aplicaciones de tintas serigráficas mediante técnicas de huecograbado y tecnología inkject y piezas con aplicaciones vía seca en forma de partículas o gránulos. Posteriormente las piezas obtenidas se caracterizaron siguiendo las normativas aplicadas habitualmente a las baldosas cerámicas, obteniendo resultados óptimos.
El desarrollo del trabajo expuesto se encuentra en plena fase de implantación industrial.

\section{CONCLUSIONES}

Se han obtenido una nueva gama de productos para la decoración de baldosas cerámicas fabricadas por monococción, que dan lugar a efectos estéticos diferenciales, caracterizados porque, tras su aplicación sobre un sustrato cerámico y posterior cocción del mismo, se genera una estructura de microgrietas en dichos recubrimientos que provoca la aparición de un efecto iridiscente, multicolor en la pieza final cuando esta es iluminada con luz blanca que confiere a la pieza cerámica dichas características estéticas diferenciales, con un aspecto similar al que se puede observar en el mineral "cuarzo iris o cuarzo rainbow".

En el presente estudio se ha realizado un análisis del estado de la técnica, y se ha profundizado en el conocimiento, estudio y caracterización de los fenómenos físico-ópticos responsables del efecto final observado en las piezas. Estos fenómenos ópticos, su estudio y comprensión, sus mecanismos de obtención y potenciación, muy utilizados en otros sectores tales como el sector de las pinturas y plásticos, pueden permitirnos abrir nuevas líneas de investigación para continuar dotando a nuestro sector de nuevas propuestas decorativas que permitan seguir diferenciando y diversificando nuestros azulejos.

El poder determinar y modelizar como se produce el "efecto multicolor" o efecto iridiscente en determinados minerales, fijar la causa de dicho efecto, determinar el fenómeno físico-óptico que lo provoca, analizar y entender los mecanísmos fenomenológicos que lo causan nos puede permitir intentar extrapolar dichos efectos a nuestros materiales cerámicos, desarrollar nuevos recubrimientos que presenten propiedades ópticas determinadas y poder dotar a nuestras decoraciones de nuevas propiedades que les confieran mayor valor añadido.

Con el desarrollo de este proyecto, se ha obtenido el conocimiento necesario para entender el fenómeno que causa el efecto multicolor y como se origina. Este conocimiento es fundamental para poder comprender las modificaciones que se pueden hacer en un material para conseguir distintos efectos a partir del mismo fenómeno, así como para conocer cuáles son los límites; es decir, bajo que condiciones es posible generar el efecto y bajo cuales no.

Se ha dotado a los productos de las características técnicas, estabilidad y reproducibilidad necesarias para su utilización en la decoración de baldosas cerámicas, en un amplio rango de curvas de cocción sin necesidad de efectuar variaciones en los ciclos de cocción habituales de la industria cerámica. A su vez se han realizado estudios para acoplar estos productos a los métodos de decoración habituales del sector cerámico. Es importante resaltar que la formación, localización y control del tamaño de las microgrietas formadas entre las partículas o gránulos y la matriz o esmalte cerámico ha sido importante, no solo para conseguir los efectos ópticos deseados, sino también para evitar que la presencia de microgrietas pueda dar lugar a bajas características técnicas de las piezas realizadas.

Estéticamente, se presentan novedosas posibilidades de decoración, dado que con los materiales desarrollados se permite la obtención del efecto iridiscente, multicolor en toda la pieza o sólo en ciertos puntos de la misma, y junto con la 
posibilidad de variación de efectos y coloraciones, permiten la realización de ilimitados tipos de modelaje.

Se abre así una nueva línea de trabajo con productos innovadores en el sector cerámico, gracias al conjunto de ventajas aportado y amplía la gama de productos y conocimientos con los que puede contar el sector, posibilitando la obtención de nuevos recubrimientos superficiales para baldosas cerámicas. De esta forma, avanzamos en el propósito de diferenciar y diversificar las posibilidades estéticas en el sector azulejero.

El proyecto presentado junto con los productos obtenidos ha sido probado industrialmente en las instalaciones de varios de nuestros clientes y dada la novedad de los resultados obtenidos con el presente trabajo, la gama de productos que se presentan han sido objeto de la solicitud de patente por parte de la empresa.

\section{BIBLIOGRÁFIA}

1. R. Uribe, "Desarrollo de materiales estructurales de alúmina titanato de aluminio con alta resistencia al choque térmico", Tesis Doctoral, Universidad Autónoma de Madrid, 2001.

2. J. B. Walsh, "Effect of cracks on the compressibility of rock," J. Geophys. Res., 70 [ 2 ] 381-389 (1965).
3. C. Baudin C. Baudín y P. Miranzo, "Caracterización de la resistencia al choque térmico de materiales frágiles," Anales de la mecánica de la fractura . 12 261-65 (1995).

4. Austin A.E. Austin and C.M. Schwartz, "The crystal structure of aluminum titanate," Acta Cryst ., 6 812-13 (1953).

5. A.G. Evans, "Microfracture from thermal expansion anisotropy-I. Single fase systems," Acta Metall ., 26 1845-53 (1978)

6. R.W. Davidge, "Cracking at grain boundaries in polycristalline brittle materials," Acta Metall., 29 1695-702 (1981).

7. J. Kuszyk y R.C. Bradt, "Influence of grain size on effects of thermal expansion anisotropy in $\mathrm{Mg} 2 \mathrm{TiO} 5$," J. Am. Ceram. Soc., 56 [ 8 ] 420-23 (1973).

8. J. Cleveland and R.C. Bradt, "Grain size/microcracking relationships for pseudobrookite oxides," J. Am. Ceram. Soc., 61 [ 11-12 ] 478-81 (1978).

9. Y. Ohya and Z. Nakagawa, "Grain-boundary microcracking due to thermal expansion anisotropy in aluminum titanate ceramics," J. Am. Ceram. Soc., 70 [ 8 ] C-184-C-86 (1987).

10. H. Wohlfromm, "Preparación por sinterización reactiva y estudio del comportamiento mecánico y térmico de materiales de titanato de aluminio," Tesis Doctoral, Facultad de Ciencias. Universidad Autónoma de Madrid, (1991).

11. R.W. Davidge, "Mechanical behaviour of ceramics" cap. The fracture streng of ceramics, pp. 75-103 (1979).

12. R.M. Cannon, R.L. Coble, "Review of diffusional creep of alumina," pp 61-100 in Deformation in Ceramic Materials, Ed. R.E. Tressler, R.C. Bradt, Plenum Press, EEUU, (1975).

13. N. Claussen, B. Mussler and M.V. Swain, "Grain-size dependence of fracture energy in ceramics," I. Am. Ceram. Soc ., 65 [ 1 ] C14-C16 (1982).

14. ASTM C 1198-91. Annual book of ASTM Standards 2000. Vol. 15.01

15. W.D. Kingery, H.K. Bowen and D.R. Uhlmann. "Thermal and compositional stresses", pp. 768-815 in "Introduction to ceramics". Ed. A Wiley-Interscience, Publication John Wiley \& Sons, USA (1976). 